\title{
S-parameter uncertainty computations
}

\author{
Vidkjær, Jens
}

\section{Published in:}

Proceedings of the 23rd European Microwave Conference

Link to article, DOI:

10.1109/EUMA.1993.336732

Publication date:

1993

\section{Document Version}

Publisher's PDF, also known as Version of record

Link back to DTU Orbit

\section{Citation (APA):}

Vidkjær, J. (1993). S-parameter uncertainty computations. In Proceedings of the 23rd European Microwave Conference (pp. 866-868). IEEE. https://doi.org/10.1109/EUMA.1993.336732

\section{General rights}

Copyright and moral rights for the publications made accessible in the public portal are retained by the authors and/or other copyright owners and it is a condition of accessing publications that users recognise and abide by the legal requirements associated with these rights.

- Users may download and print one copy of any publication from the public portal for the purpose of private study or research.

- You may not further distribute the material or use it for any profit-making activity or commercial gain

- You may freely distribute the URL identifying the publication in the public portal

If you believe that this document breaches copyright please contact us providing details, and we will remove access to the work immediately and investigate your claim. 


\section{S-parameter uncertainty computations}

\section{J.Vidkjær}

Electromagnetics Institute, Tech. Univ. of Denmark, DTH bldg. 348 , DK2800 Lyngby, Denmark, Tel:+45 42 881444; Fax:+45

$931634 ;$ e-mail jv@sysmns.emi.dth.dk

\section{Abstract}

A method for computing uncertainties of measured sparameters is presented. Unlike the specification software provided with network analyzers, the new method is capable of calculating the uncertainties of arbitrary s-parameter sets and instrument settings.

\section{Introduction}

The uncertainties on measured s-parameters are valuable information in their own rights, but particularly in conjunction with modelling tasks. Here the accuracy of the experimental foundation behind a model may guide the fitting process and constitute an objective criterion, possibly the only one, for estimating the quality of the result. This usage of uncertainties has proven to be advantageous and has been reported elsewhere, Vidkjær et al. (1),(2). The present paper concentrates on how the uncertainties are actually calculated from three types of information, the accuracy specification of the network analyzer, its operating condition in the particular set of calibrations and measurement, and the s-parameter values themselves.

The reason why the present work was necessary are the two last items above. Accuracy specifications on network analyzers are generally not available in a form which makes it possible directly to calculate uncertainties corresponding to all the situations which occur in measurements for device modelling. As a matter of fact, such specifications have evolved from being reasonably accessible, although incomplete, in the forms of graphs, tables, and formulas in low frequency and older models, HP8753C, HP8510A to becoming completely opaque in HP8510B and gradually improving with the HP8510C network analyzers. In any case, however, specifications apply to only one setting of the power level which may be too high to guarantee undisturbed measurements for precision modeling of transistors. Moreover, they often apply only to a limited set of s-parameters which bears no resemblance to active device data; e.g. in $s_{11}$ and $s_{22}$ measurements $s_{21}$ and $s_{12}$ are commonly assumed zero and visa versa.

\section{Basic Uncertainty Computations}

The method to be presented has two major ingredients. An updating algorithm for calculating the resultant s-parameter uncertainties from the various contributions and a model of the dynamic errors in the receiver part of the network analyzer. The latter is used for generating or adjusting known error contributions to the power levels, which are actually applied.

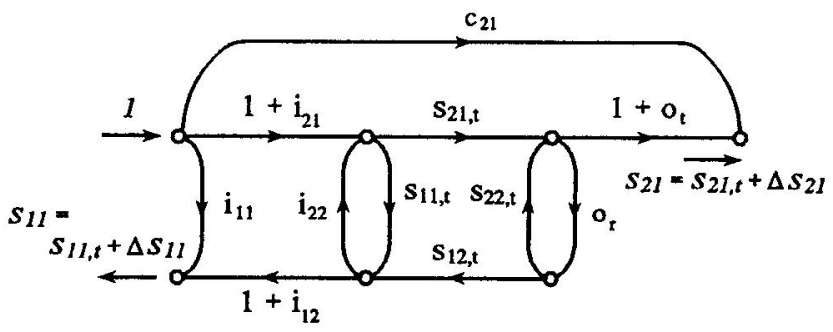

Below, we consider first the algorithm and a few fundamental concepts which are essential to its formulation and utilization. In order to concentrate on principal aspects, no distinction is made between magnitude and phase errors, as they both follow the same computational schemes.

A flowgraph for the residual errors in a forward direction sparameter measurement is shown in Fig. 1, where the relative errors, $i_{11}, i_{12}, i_{21}, i_{22}$, or, ot, and $c_{12}$, are all assumed small compared to one. Thereby all 2 nd order terms in these errors may be ignored, and the absolute s-parameter uncertainties may be calculated from the flowgraph to yield,

$$
\begin{aligned}
& \Delta s_{11}=i_{11}+i_{12}\left|s_{11}\right|+i_{21}\left|s_{11}\right|+i_{22}\left|s_{11}\right|^{2}+o_{r}\left|s_{12}\right|\left|s_{21}\right| \\
& \Delta s_{21}=i_{21}\left|s_{21}\right|+i_{22}\left|s_{11}\right|\left|s_{21}\right|+o_{r}\left|s_{22}\right|\left|s_{21}\right|+o_{\imath}\left|s_{21}\right|+c_{21}
\end{aligned}
$$

As a consequence of the assumption of small errors, it should be realized, that if a flowgraph of the type in Fig. 1 is embedded in a similar error graph, the resultant uncertainties would be equal to the results we get by collecting equivalently placed errors in a single graph. Therefore, more detailed error graphs than Fig. 1, e.g. the error models accompanying instrument specifications, (3),(4), may illustrate error origins, but they serve no additional computational purposes. Eqs. (1) and (2) are linear in the error terms, $i_{11}, i_{12}, \ldots$, so it is not necessary first to include all contributions to the branches in the error graph before the equations are used. This would be required for setting up closed form uncertainty formulas of the types given in (3) and (4), but here an algorithmic updating approach is taken.

The updating process is illustrated by Fig. 2. As input we keep the list of all different errors like residual directivities, load matches, connector repeatabilities etc. They are given a primary classification of where in the error model they contribute, that is one or more of the placements i11 through c21. When the list is scanned, the corresponding s-parameter uncertainty contributions are found from Eqs. (1),(2) and accumulated. As indicated in the figure, each accumulating register is subdivided in two directions, by the nature of the error, which is given through the input list, and by the accumulation method, worst case (WCS) or RMS.

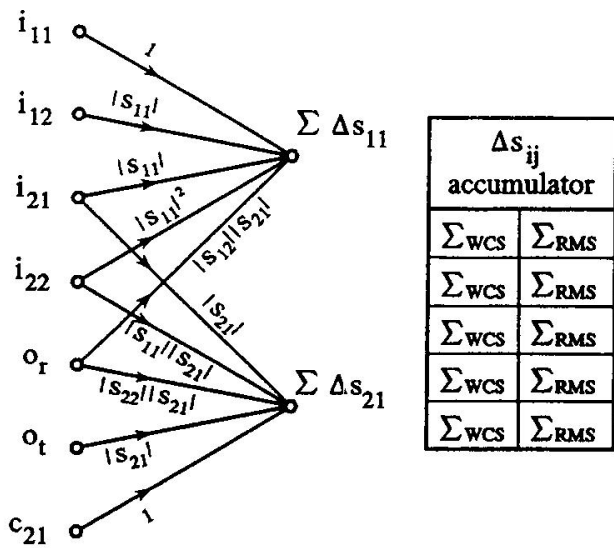

systematic errors drift errors repeatability errors measurement noise calibration noise

Fig.1. Flowgraph for residual errors in forward measurements. $s_{\mathrm{ij}, \mathrm{t}}$ represents true s-parameters. 
In network analyzer literature and specifications it is common to distinguish between the error types, (4),

a) systematic errors

b) drift errors

c) random errors from switch and connector repeatabilities

d) random errors from electrical noise

When more errors are combined, systematic errors are added to give a worst case result, while drift and random errors are combined like noise in an RMS sense. It is supposed that this choice most directly suits the purpose of calculating guaranteed performance specifications. For the sake of setting up statistical foundations for modelling tasks, however, other ways of combining errors may be preferable. Following the discussion in C.F. Dietric (5), the distinction between terms to be updated worst case or RMS should be made on whether or not they are correlated, regardless of their systematic or random nature.

One advantage of the updating scheme in Fig. 2 compared to closed form expressions is, that there are made no initial assumptions on how errors are combined. This depends upon the operation of the updating registers in the actual computation. Error types which are chosen to be updated worst case are added to their WCS accumulator. Error types chosen to be RMS updated are firstly accumulated in WCS mode. When all contributions from a group of correlated errors are collected, which as a minimum includes all possible different paths through the graph of Fig. 2 from one single error origin, the result is squared and transferred to the corresponding RMS accumulator. When all inputs are done, the resultant s-parameter uncertainties are found by summing across the type accumulators taking the square root of the final RMS result.

It should be noted that compared to the list of error types above, the algorithm distinguishes between errors originating from noise in measurements and in calibrations. The separation is made by the dynamic accuracy model described below. By keeping track of errors from electrical noise in both measurements and calibrations, it is possible to incorporate the effect of using different IF bandwidths and/or averaging counts between measurements, calibrations and specifications. The squared RMS noise grows proportional to bandwidth and inversely proportional to the averaging count, (5), and the updating accumulators may be scaled accordingly. It is a second advantage of this method, that in addition to the total uncertainties of each s-parameter, its statistical components are recorded, so the consequences of different measurement strategies may be visualized.

\section{Dynamic Accuracy}

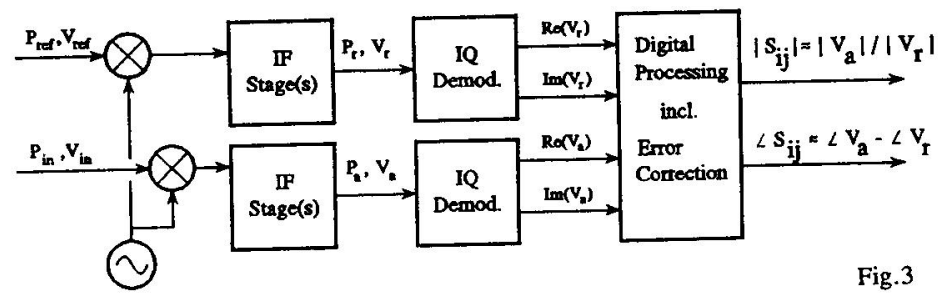

Receiver principle for detection of one s-parameter (HP8510B). The output expressions are exact if no error corrections were required.

Dynamic errors are caused by nonlinearities, especially compression, and noise in the receiver circuits of the network analyzer. In order to make uncertainty calculations for other power levels than those assumed in the original uncertainty specifications, these effects must be described as functions of the input levels to the receiver. We shall here outline the essence of a procedure for deriving the functions. Consider first the transmission through the initial mixer and IF stages of a single channel in a receiver, Fig. 3 . It may be written,

$$
V_{a}=G\left(V_{i n}\right) V_{i n}+V_{d v}=G_{i n} V_{i n}\left(1+\Delta g\left(V_{i n}\right)+\frac{V_{w s}}{G_{i n} V_{i n}}\right)
$$

where $G\left(V_{i n}\right)$ is the nonlinesur, deterministic transfer function and $V_{n f}$ represents the noise floor. In the second expression the two last terms inside the parenthesis give the relative gain compression, $\Delta \mathrm{g}$ and the relative noise level in a single channel with reference to a linear but yet unknown gain $\mathrm{G}_{\mathrm{in}}$. Taking the ratio between the outputs from the two channels in the receiver, assuming that $\Delta \mathrm{g}$ and the relative noise are small compared to one and that the two channels are similar, gives a raw, uncorrected s-parameter deternination,

$s_{\text {rew }}=\frac{V_{c}}{V_{\text {ref }}}=\frac{G_{\text {in }}}{G_{r e f}}\left(1+\Delta g\left(V_{i n}\right)-\Delta g\left(V_{r e f}\right)+\sqrt{\frac{V_{n j}^{2}}{G_{i n}^{2} V_{i n}^{2}}+\frac{V_{N f}^{2}}{G_{r e f}^{2} V_{r e f}^{2}}}\right)$.

By calibrating, we make in principle a reference for the result by a similar raw measurement $V_{a, c a l} / N_{\text {ref,eal }}$ on a known standard, for instance $s=1$ when calibrating with a thru. This fixes the linear gains and provides the final corrected result,

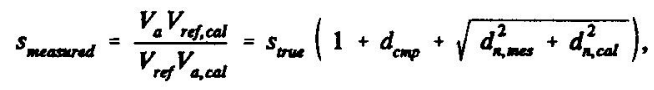

where error contributions from compression, $d_{c m p}$, measurement noise, $d_{n, \text { mes}}$, and calibration, $d_{n, c a l}$, become,

$$
\begin{aligned}
& d_{c m p}=\left|\Delta g\left(V_{i n}\right)-\Delta g\left(V_{i n, \text { cel }}\right)-\Delta g\left(V_{\text {ref }}\right)+\Delta g\left(V_{\text {ref,cat }}\right)\right|, \\
& d_{n \text { mess }}=\sqrt{\frac{V_{v f}^{2}}{G_{i n}^{2} V_{i n}^{2}}+\frac{V_{v}^{2}}{G_{r e f}^{2} V_{r e f}^{2}}}, \quad d_{\text {ceal }}=\sqrt{\frac{V_{d}^{2}}{G_{i n}^{2} V_{i n, c a l}^{2}}+\frac{V_{V}^{2}}{G_{r d f}^{2} V_{r e f, c a l}^{2}}}
\end{aligned}
$$

This is the type of expressions we are seeking, provided that the noise constants and the $\Delta \mathrm{g}$ function may be identified. Considering the noise terms, they show the characteristic asymptote of 1 decade rise in error per $20 \mathrm{~dB}$ reduction of input level present in all dynami, error specifications, so under the assumption that all channels, are approximately equal, the $V_{n f} / G_{\text {in }}$ $=V_{n f} / G_{\text {ref }}$ are easily recognized, if they are not directly a part of the specifications. A complete dynamic accuracy specification includes both the compression and the noise terms. Stripping off the now known noise isolates the compression error in Eqi. (6). As data are commonly given using $V_{\text {ref }}=V_{\text {ref,eal, }}$, the two last terms cancel. With exception of an unimportant constant and sign, the $\Delta \mathrm{g}$ function may now be reconstructed from the remaining $\mathrm{d}_{\mathrm{cmp}}$ term based on its distinct minimum at $\mathrm{V}_{\text {in }}=\mathrm{V}_{\text {in,cal }}$.

The usage of the dynamic error expressions above in the total uncertainty computation s twofold. The errors in an actual measurement may be calculated and combined directly by adding additional inputs to the updating scheme in Fig. 2. Calibration errors, on the other hand, are replacements of similar, but not separately specified terms in the original error data. Knowing the input levels where the original data apply, the equations above are used first to calculate the corresponding calibration errors and then to insert only the differences to the similar error at the new input levels. 


\section{Discussions and Conclusions}

The presentation above emphasized a few key aspects in the formulation of algorithms for computing uncertainties of sparameters. A huge amount of important details were left out, for instance on the inclusion of trace noise and autoranging gain errors, the scaling of calibration error replacements, or the calculation of receiver input levels in measurements and calibrations. They are available in Vidkjær (6), which also describes the specific implementation of the methods for the HP8753C and HP8510B network analyzers. The capabilities of the resultant program, a $\mathrm{C}$ function, may be summarized by Table 1 . Here all operating conditions are user definable within the limitations of the specific instrument, and the program calculates the corresponding uncertainties for any set of s-parameters. Especially in device measurement for modelling tasks, the flexibility of the new program compared to common uncertainty specifications has proven highly useful.

\section{References}

(1) J. Vidkjær, "Accuracy bounds in Small-Signal Model Identifications", Proc. Europ. Conf. - Circt. Theory and Design, 1991, pp. 782-791.

(2) J. Vidkjær, V. Porra, J. Zhu, T. Huttunen, "Validation of Nonlinear Bipolar Transistor Model by Small-Signal Measurements", Proc. 22nd Europ. Microw. Conf., 1992, pp 12171222.

(3) HP8753C Network Analyzer, Operating Manual, Hewlett Packard, part no. 08763-90155, May 1989, General Information section.

(4) HP8510C On-Site Service Manual, Hewlett Packard, part no. 08510-90282, May 1991, Performance Verification and Specifications section.

(5) C.F. Dietrich, "Uncertainty, Calibration and Probability, The Statistics of Scientific and Industrial Measurements", Adam Hilger, Bristol, 2nd ed. 1991

(6) J. Vidkjær, "Network Analyzer Uncertainty Computations", Lab. Report R549, Electromagnetics Inst., Tech. Univ. Denmark, Aug. 1993.

Table 1. Example of network analyzer configuration and operation conditions.

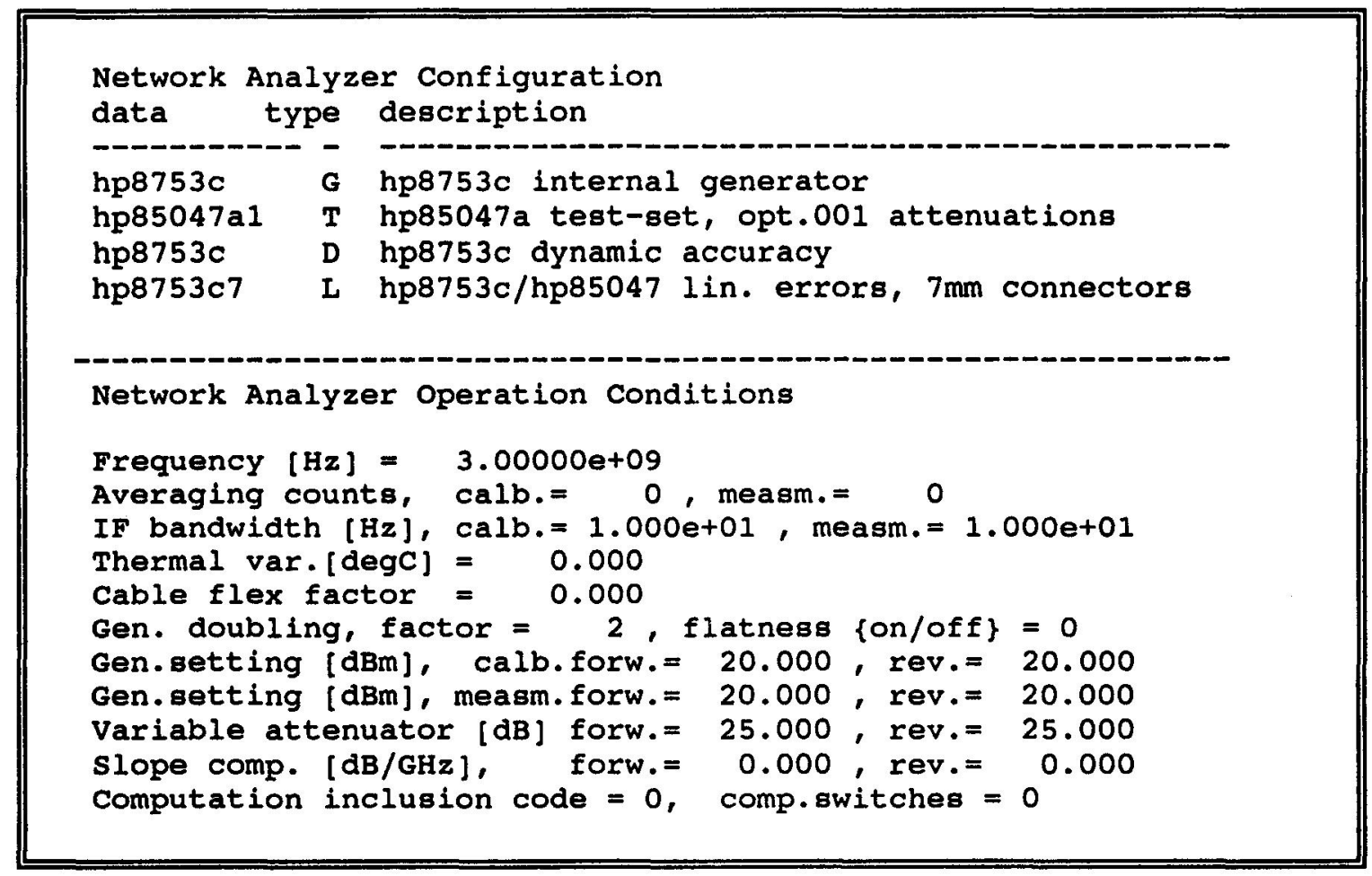

